\title{
Effects of Diluents on the Separation of Minor Actinides from Lanthanides with Tetradodecyl-1,10-phenanthroline-2,9-diamide from Nitric Acid Medium
}

\author{
Nao TsutsuI,,$* * \dagger$ Yasutoshi BAn, ${ }^{* *}$ Hideya SuzukI, $* *$ Masahiko NAKaSe, ${ }^{*}$ Sayumi Ito, $*$ \\ Yusuke InABa, $*$ Tatsuro MatSumura, $* *$ and Kenji TAKeShita* \\ *Institute of Innovative Research, Tokyo Institute of Technology, 2-12-1-N1-16 Ookayama, Meguro, \\ Tokyo 152-8550, Japan \\ **Research Group for Partitioning, Japan Atomic Energy Agency, 2-4 Sirakata, Tokai, Ibaraki 319-1195, Japan
}

\begin{abstract}
To investigate the effective separation of actinides (Ans) from lanthanides (Lns), single-stage batch extraction experiments were performed with a novel extractant, tetradodecyl-1,10-phenanthroline-2,9-diamide (TDdPTDA) with various diluents such as 3-nitrobenzotrifluoride (F-3), nitrobenzene, and $n$-dodecane for Am, Cm, and Lns. The extraction kinetics with TDdPTDA was rapid enough to perform continuous extraction experiments using mixer-settler extractors. The slopes of the distribution ratio versus the TDdPTDA concentration and the distribution ratio versus the nitric acid concentration were similar for F-3 and nitrobenzene systems, but different from the $n$-dodecane system. These differences were attributed to the characteristics of the diluents. This study revealed high distribution ratios of $\mathrm{Am}\left(D_{\mathrm{Am}}\right)$ and $\mathrm{Cm}\left(D_{\mathrm{Cm}}\right)$ for TDdPTDA, with the high separation factors $(S F \mathrm{~s})$ of Am from Lns enough for their separation.
\end{abstract}

Keywords Partitioning, 1,10-phenanthroline-2,9-dicarboxamide, minor actinide, americium, curium, lanthanide, solvent extraction, distribution ratio, separation factor

(Received July 23, 2019; Accepted September 19, 2019; Advance Publication Released Online by J-STAGE September 27, 2019)

\section{Introduction}

The treatment of high-level liquid waste (HLLW) generated by the reprocessing of spent nuclear fuels is important for sustainable development involving nuclear energy. To reduce the radiotoxicity and heat generation of HLLW and the volume of radioactive waste, technology related to Partitioning and Transmutation (P \& T) has been investigated. ${ }^{1-3}$ In Partitioning, the HLLW is initially separated based on the chemical properties of its constituents. This is followed by the separation of the long-lived minor actinides (MAs) like $\mathrm{Np}, \mathrm{Am}$, and $\mathrm{Cm}$, and transmutation by fast reactors or accelerator driven systems. Thus, the development of an effective partitioning method of MAs from spent nuclear fuel is necessary for efficient transmutation. Extensive studies on partitioning exist and several hydrometallurgical processes including SANEX (Selective Actinide Extraction), ${ }^{4}$ DIAMEX (Diamide extraction), ${ }^{5}$ TALSPEAK (Trivalent Actinide Lanthanide Separation by Phosphorus-reagent Extraction from Aqueous Komplexes), ${ }^{6}$ LUCA (Lanthaniden Und Curium Americum Trennung), ${ }^{7}$ and ALSEP (Actinide Lanthanide Separation) ${ }^{8}$ have been proposed.

The intra- and intergroup separation of trivalent lanthanides (Lns) and trivalent actinides (Ans) is among the most challenging separations of metal ions in the nuclear field. The very large neutron absorption cross sections of some Lns causes them to

$\dagger$ To whom correspondence should be addressed.

E-mail: tsutsui.nao@jaea.go.jp act as neutron poisons that interfere with chain reactions, sustain the criticality by neutron absorption and lowering the efficiency of the transmutation process. Therefore, the efficient separation of Ans from Lns is critical. Since Lns are stable in the trivalent state, the separation of light Ans (e.g., Th, U, Pu, and $\mathrm{Np}$ ) from Lns is possible through modification of the oxidation states of Ans to the tetravalent and/or hexavalent state. ${ }^{9-11}$ The separation of $\mathrm{Am}(\mathrm{III})$ and $\mathrm{Cm}(\mathrm{III})$ from $\mathrm{Ln}(\mathrm{III})$ is, however, difficult because of the similarity in the chemical properties. We introduced several extractants consisting of carbon, hydrogen, oxygen, and nitrogen atoms (so-called $\mathrm{CHON}$ principle) to recover MAs for transmutation, and proposed a hydrometallurgical separation process, named SELECT (Solvent Extraction from Liquid-waste using Extractants of CHON-type for Transmutation). ${ }^{3}$ This process consists of the following four steps: (i) the recovery of $\mathrm{U}$ and $\mathrm{Pu}$ from the dissolution solution of spent nuclear fuels, (ii) the recovery of $\mathrm{Am}, \mathrm{Cm}$, and Lns from HLLW, (iii) the mutual separation of Lns from Am and $\mathrm{Cm}$, and (iv) the separation of Am with $\mathrm{Cm}$. In these, last two steps are very important, but also challenging. Although we proposed $N, N, N^{\prime}, N^{\prime}, N^{\prime \prime}, N^{\prime \prime}$-hexaoctyl nitrilotriacetamides $(\text { HONTA })^{12,13}$ and alkyl diamide amine (ADAAM) $)^{14,15}$ as extractants for steps (iii) and (iv), respectively, the exploration of more suitable extractants for these steps continues to optimize the separation performance of the SELECT process. Although these extractants have excellent extraction properties, there are disadvantages to be overcome. HONTA, for example, has a low distribution ratio for $\mathrm{Am}$ and $\mathrm{Cm}$ under highly acidic condition, and ADAAM hardly extracts $\mathrm{Cm}$. Therefore, it is important to 


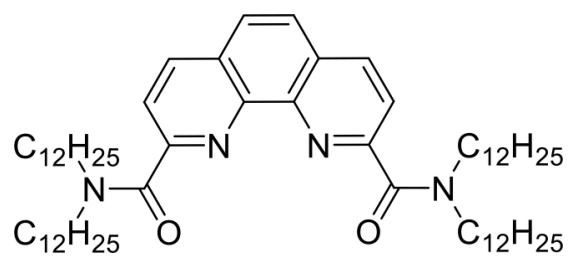

Fig. 1 Molecular structure of tetradodecyl-1,10-phenanthroline-2,9diamide (TDdPTDA).

explore more suitable extractants.

Studies on MA/Ln separation are common and, recently, the combination of $\mathrm{N}$ and $\mathrm{O}$ donors in a ligand emerged and is attracting significant attention. A soft $\mathrm{N}$-donor coordinates preferentially with $\mathrm{Am}$ and $\mathrm{Cm}$ instead of Lns because of the slightly softer characteristics of the former, including empirical rules of Hard and Soft Acids and Bases theory. However, the easy protonation of extractants comprising $\mathrm{N}$-donor atoms restricts the use of such extractants under highly acidic conditions. Contrarily, extractants containing hard O-donors show high affinity toward An and Ln and operate in a highly acidic condition. Among these extractants, the 1,10-phenanthroline2,9-dicarboxamides (PTDAs), containing the phenanthroline structure with soft $\mathrm{N}$-donors and hard O-donors, showed a high coordination ability toward Ans. ${ }^{11,16}$ Alyapyshev et al. synthesized PTDAs with different alkyl groups and evaluated their extraction abilities for Am, Ln, and other metals like $\mathrm{Cd}$, $\mathrm{Pb}$, and $\mathrm{Cu} .{ }^{17}$ They found that the TBuPhen in polar diluents, like 3-nitrobenzotrifluoride (F-3), demonstrated excellent extraction and separation performance for $\mathrm{Am} / \mathrm{Eu}$. Galletta et al. reported PTDA derivatives and a high distribution ratio of $\mathrm{Am}$ and the separation performance of Am from $\mathrm{Eu}$ were reported with $o$-nitrophenyl hexyl ether (NPHE) and brominated cobalt bis(dicarbollide) anion (Br-Cosan) as a diluent and synergist. ${ }^{9}$ Structural and thermodynamic studies on PTDAs with light Ans including $\mathrm{U}$ and Th, and some Lns were also reported, accompanied by the determination of bond lengths of the metal $\mathrm{O}$ and $\mathrm{N}$ and stability constants. ${ }^{18,19}$ Some studies concerning chemical calculation based on density functional theory (DFT) has also been conducted, and the structures of complexes were examined, ${ }^{9}$ and the stability of PTDA complexes was rationalized. ${ }^{18}$

In this study, we proposed tetradodecyl-1,10-phenanthroline2,9-diamide (TDdPTDA, Fig. 1) as an extractant for MA/Ln separation. Four dodecyl groups were introduced to increase the molecular solubility in $n$-dodecane. This study also investigated the general extraction behavior of a series of Lns and MAs. Moreover, separation factors of An toward Ln, which is desirable to exceed five for practical implementation, were evaluated and discussed.

\section{Experimental}

\section{Reagents}

TDdPTDA was purchased at Chemicrea, Inc. (Tokyo, Japan), while the radioactive isotopes ${ }^{152} \mathrm{Eu},{ }^{241} \mathrm{Am}$, and ${ }^{244} \mathrm{Cm}$ were purchased from Japan Radioisotope Association (Tokyo, Japan). Other reagents were of analytical grade and used without further purification.

\section{Procedure of batch extraction experiments}

The organic phases involved the dilution of TDdPTDA with
F-3, nitrobenzene, $n$-dodecane, $o$-nitrophenyl octyl ether (NPOE), and toluene. When $n$-dodecane was used as the diluent, 10 vol.\% 2-ethyl-1-hexanol was added to prevent the formation of a third phase immiscible in the aqueous and organic phases. Each organic phase was pre-equilibrated using identical concentrations of nitric acid before extraction experiments. The aqueous phases comprised 0.2 to $10 \mathrm{M}$ of nitric acid with trace concentrations of ${ }^{152} \mathrm{Eu}$ (representing of Lns), ${ }^{241} \mathrm{Am}$, and ${ }^{244} \mathrm{Cm}$ (for RI extraction) or $1.0 \mathrm{mg} / \mathrm{dm}^{3}$ of $14 \mathrm{Lns}$ (from $\mathrm{La}$ to $\mathrm{Lu}$ expect for Pm, for Ln extraction). Equal volumes $\left(1.6 \mathrm{~cm}^{3}\right.$ for the RI experiment and $1.0 \mathrm{~cm}^{3}$ for the $\mathrm{Ln}$ experiment) of organic and aqueous phases were vigorously mixed in a glass vial with a screw cap using a shaker (ModelYS-8D, YAYOI) for 0.5 to $60 \mathrm{~min}$ at room temperature ( $293 \pm 2 \mathrm{~K})$. The mixtures were separated into two phases using a centrifuge (CN-1050, ASONE), and both phases were sampled individually.

A $0.5-\mathrm{cm}^{3}$ portion of each aqueous phase was diluted with $2.5 \mathrm{~cm}^{3}$ of $0.1 \mathrm{M}$ nitric acid in a $6-\mathrm{cm}^{3}$ vial. A $0.5-\mathrm{cm}^{3}$ portion of each organic phase was diluted with $2.5 \mathrm{~cm}^{3}$ of 2-ethyl-1hexanol in a $6-\mathrm{cm}^{3}$ vial. The concentrations of ${ }^{152} \mathrm{Eu}$ and ${ }^{241} \mathrm{Am}$ in these samples were measured by a gamma-ray counter with a germanium detector (LO-AX 51370/20-P, ORTEC).

The concentrations of ${ }^{244} \mathrm{Cm}$ were determined by an alpha-ray counter with silicon semiconductor detectors (Alpha Ensemble, Seiko EG \& G). The samples for the analysis were prepared by the following procedure. Each $0.1 \mathrm{~cm}^{3}$ portion of sample was diluted with $0.9 \mathrm{~cm}^{3}$ of ethanol, and a portion of each diluted sample was placed on a stainless-steel disk. The sample on the disk was dried by an infrared lamp, and then heated by an electric furnace. Three disks were prepared for each sample for triplicate measurements.

In an experiment involving $14 \mathrm{Lns}$, both phases were separated by a centrifuge (4000, KUBOTA) and then, a $0.4-\mathrm{cm}^{3}$ portion of each organic phase was vigorously mixed with a $4.0-\mathrm{cm}^{3}$ stripping solution containing of $0.1 \mathrm{M}$ tetraethyldiglycolamide (TEDGA) in a glass vial with a screw cap to back-extract Lns into the stripping solution. The concentrations of the $14 \mathrm{Lns}$ in the aqueous and the back-extracted solutions were measured by ICP-MS (7500, Agilent). A standard solution, XSTC-1 (SEISHIN, Japan), which contained 14 Lns, was diluted with $0.1 \mathrm{M}$ nitric acid, and solutions with concentrations of $0.01,0.1$, $1.0,10,20 \mathrm{mg} / \mathrm{dm}^{3}$ for the $14 \mathrm{Lns}$ were prepared. Calibration curves for the $14 \mathrm{Lns}$ were obtained from these solutions.

The distribution ratios and the separation factor were calculated from the following equations

$$
\begin{aligned}
& D_{\mathrm{M}}=\frac{A_{\text {org. }}}{A_{\text {aq. }}}, \\
& D_{\mathrm{M}}=\frac{C_{\text {org. }}}{C_{\text {aq. }}}, \\
& S F_{\mathrm{M}_{1} / \mathrm{M}_{2}}=\frac{D_{\mathrm{M}_{1}}}{D_{\mathrm{M}_{2}}},
\end{aligned}
$$

where $\mathrm{M}, D, A, C$, and $S F$ represent the chemical symbols of a metal, the distribution ratio of a metal ion $\mathrm{M}$, radioactivity, concentration, and separation factor, respectively. The subscripts org. and aq. represent the organic and aqueous phases, respectively.

\section{Results and Discussion}

Batch experiments were conducted to investigate the time 
required to attain extraction equilibrium. Figure S1 (Supporting Information) displays the contact time dependency of $D_{\mathrm{Eu}}, D_{\mathrm{Am}}$, and $D_{\mathrm{Cm}}$ for the diluent $n$-dodecane. $D_{\mathrm{Eu}}, D_{\mathrm{Am}}$, and $D_{\mathrm{Cm}}$ at $0.5 \mathrm{~min}$ were $0.048,0.28$, and 0.13 , respectively, with no remarkable changes observed subsequently. This indicates that the extractions of $\mathrm{Eu}, \mathrm{Am}$, and $\mathrm{Cm}$ by TDdPTDA attained equilibrium in less than $0.5 \mathrm{~min}$. Previous experiments using mixer-settler extractors with monoamides as extractants showed that a few minutes of the contact were enough to exhibit efficient separation performance. ${ }^{20}$ Therefore, the extraction kinetics of TDdPTDA seems to be adequately rapid for the extraction of $\mathrm{Am}$ and $\mathrm{Cm}$ using mixer-settler extractors.

The effect of diluents on $D_{\mathrm{Ln}}, D_{\mathrm{Am}}$, and $D_{\mathrm{Cm}}$ for $0.2 \mathrm{M}$ TDdPTDA with respective diluents and $3.0 \mathrm{M}$ nitric acid is illustrated in Fig. 2. A local minimum at $\mathrm{Gd}$ was detected in each lanthanide pattern. The plots for F-3 and nitrobenzene show higher $D$ s for light Lns relative to heavy Lns, whereas plots with toluene and $n$-dodecane as diluent display higher $D \mathrm{~s}$

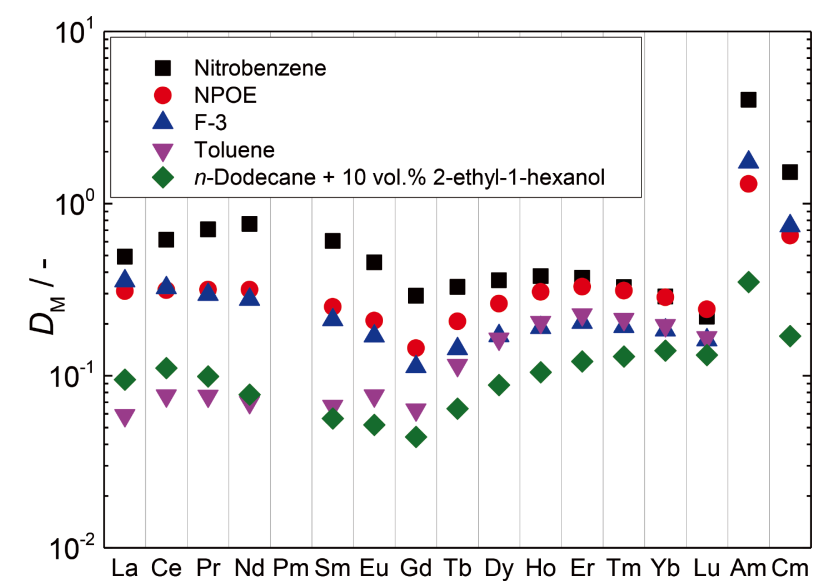

Fig. 2 Effect of diluents on $D_{\mathrm{Ln}}, D_{\mathrm{Am}}$, and $D_{\mathrm{Cm}}$ for $0.2 \mathrm{M}$ TDdPTDA diluted with respective diluents and $3.0 \mathrm{M}$ nitric acid. for heavy Lns relative to light Lns or similar values. The stability of the extraction species with poor lipophilicity in a polar diluent has been previously reported, with high lipophilicity required to maintain the stability in non-polar diluents. ${ }^{21}$ The dielectric constants were introduced as an indicator of lipophilicity and compared with each distribution ratio (Table 1). The value of $D_{\mathrm{Eu}}$ increased in the order: $n$-dodecane $+10 \mathrm{vol} . \%$ 2-ethyl-1-hexanol $<$ toluene $<$ F-3 $<$ NPOE $<$ nitrobenzene with increasing dielectric constants. Similarly, the value of $D_{\mathrm{Am}}$ also increased with increasing dielectric constant, and the tendency observed in each Lns is consistent with previous studies. ${ }^{22,23}$

The relationship between $D_{\mathrm{M}}(\mathrm{M}: \mathrm{Ln}, \mathrm{Am}$, and $\mathrm{Cm})$ and the TDdPTDA concentrations in F- 3 , nitrobenzene, and $n$-dodecane with $3.0 \mathrm{M}$ nitric acid are displayed Figs. 3(a)-3(c). Lanthanum, $\mathrm{Nd}, \mathrm{Gd}$, and $\mathrm{Lu}$ were selected as a light Ln, a chemical analog of Am, a middle Ln, and a heavy Ln, respectively. In the case of F-3 (Fig. 3(a)), $D_{\mathrm{Ln}}, D_{\mathrm{Am}}$, and $D_{\mathrm{Cm}}$ increased with increasing TDdPTDA concentration from 0.02 to $0.4 \mathrm{M}$, with the slopes for $\mathrm{Am}$ and $\mathrm{Cm}$ being 1.0 and 1.1, respectively. The slopes for $\mathrm{La}, \mathrm{Nd}, \mathrm{Eu}, \mathrm{Gd}$ and $\mathrm{Lu}$ were 1.3, $1.1,1.0,1.1$, and 1.4, respectively, these values were almost the same or slightly higher than those of Am and $\mathrm{Cm}$. These results

Table 1 Comparison of the dielectric constants of each diluent with $D_{\mathrm{Eu}}$, and $D_{\mathrm{Am}}$ for $0.2 \mathrm{M}$ TDdPTDA and $3.0 \mathrm{M}$ nitric acid

\begin{tabular}{lccc}
\hline \multicolumn{1}{c}{ Diluent } & Dielectric constant & $D_{\text {Eu }}$ & $D_{\text {Am }}$ \\
\hline Nitrobenzene & $34.75^{\mathrm{a}}$ & 0.46 & 4.01 \\
NPOE & $23.1^{\mathrm{b}}$ & 0.21 & 1.30 \\
F-3 & $22.3^{\mathrm{c}}$ & 0.17 & 1.74 \\
Toluene & $2.38^{\mathrm{a}}$ & 0.08 & - \\
2-Ethyl-1-hexanol & $7.7^{\mathrm{c}}$ & 0.05 & 0.35 \\
$n$-Dodecane & $2.012^{\mathrm{d}}$ & & \\
\hline
\end{tabular}

a. Ref. 23.

b. Ref. 25.

c. Ref. 26.

d. Ref. 27.

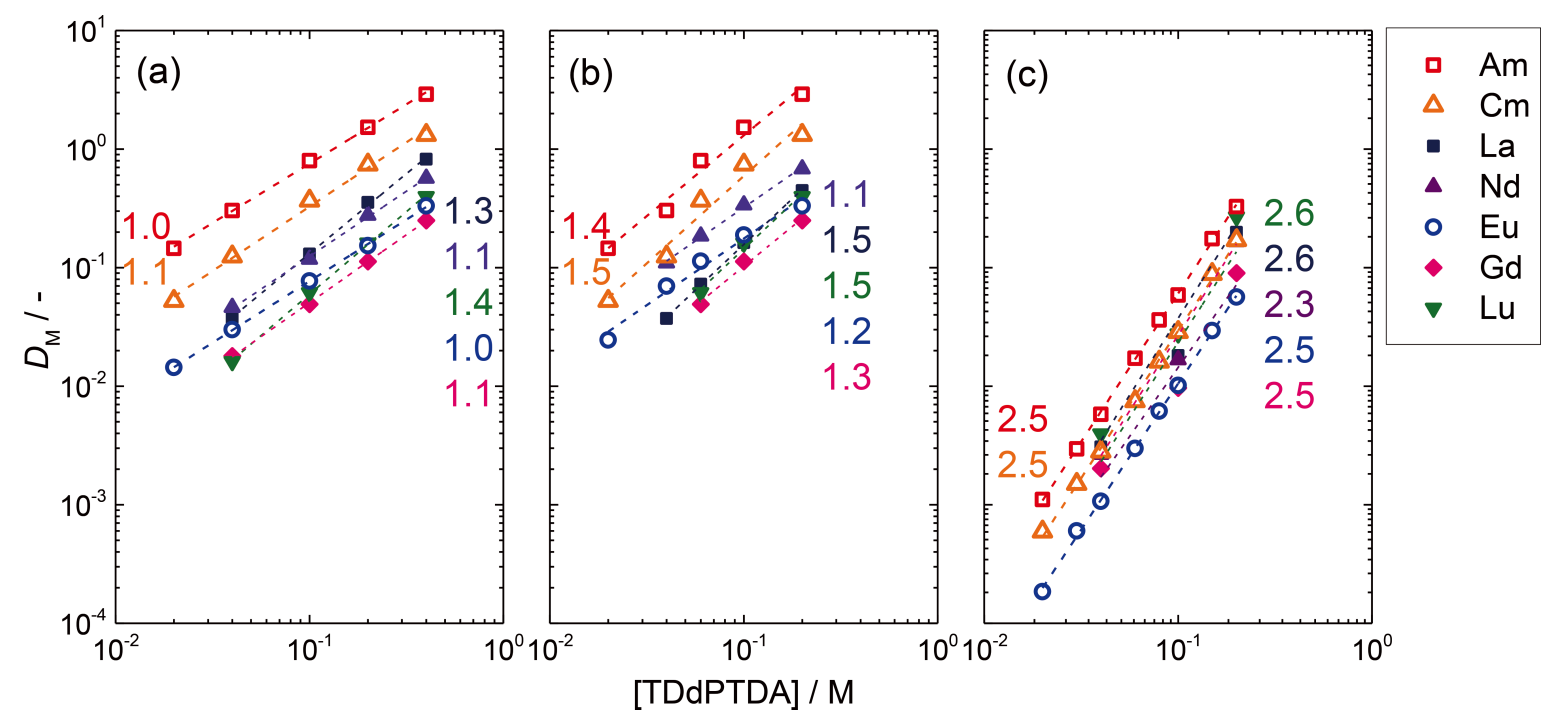

Fig. 3 Relationship between $D_{\mathrm{Ln}}, D_{\mathrm{Am}}$, and $D_{\mathrm{Cm}}$ and the ligand concentration with (a) F-3, (b) nitrobenzene, and (c) $n$-dodecane +10 vol.\% 2-ethyl-1-hexanol as the diluents, respectively. The concentration of nitric acid was $3.0 \mathrm{M}$ and the contact time was $3 \mathrm{~min}$. The numbers in the figures represent the slopes. 


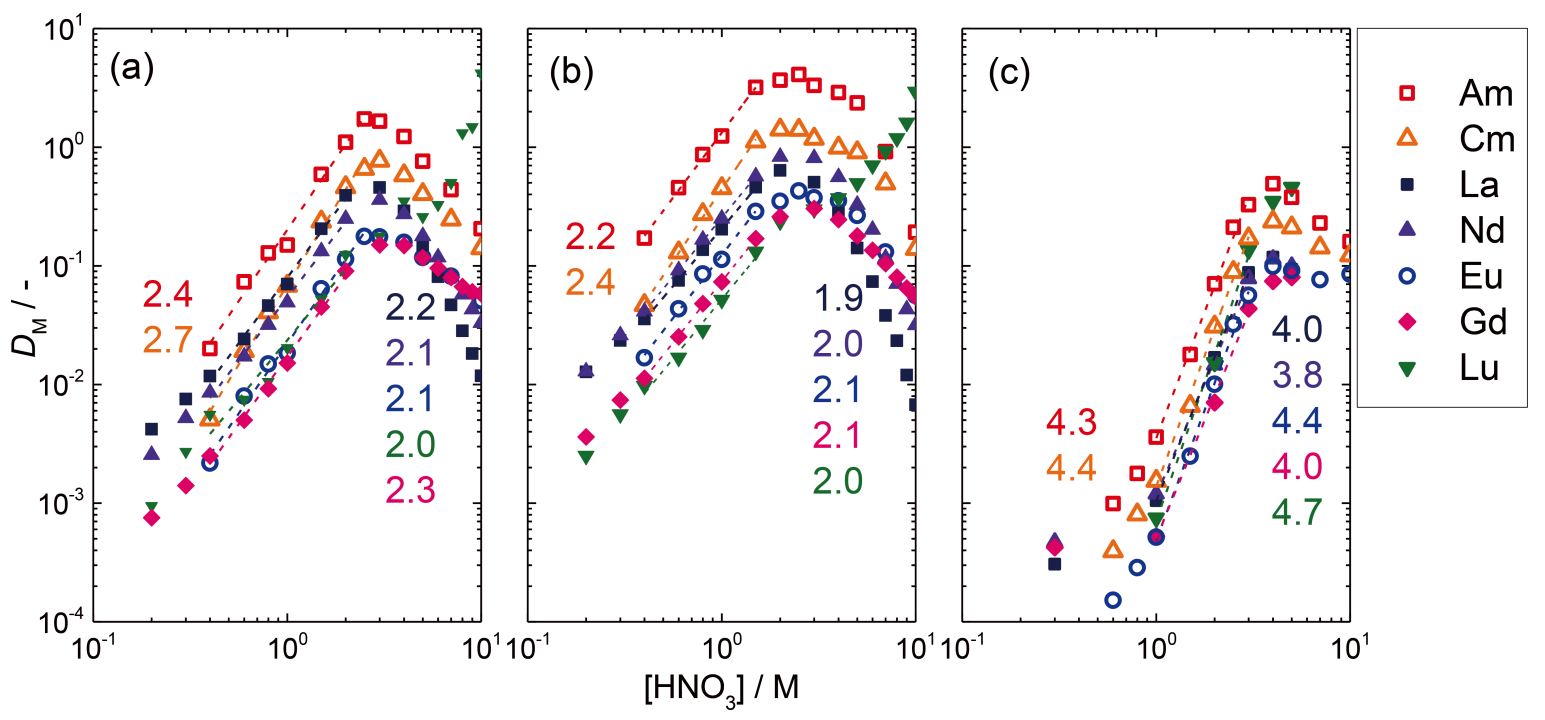

Fig. 4 Relationship between $D_{\mathrm{Ln}}, D_{\mathrm{Am}}$, and $D_{\mathrm{Cm}}$ and the nitric acid concentration with (a) F-3, (b) nitrobenzene, and (c) $n$-dodecane +10 vol.\% 2-ethyl-1-hexanol as the diluents, respectively. The concentration of TDdPTDA was $0.2 \mathrm{M}$ and the contact time was $3 \mathrm{~min}$. The numbers in the figures represent the slopes.

suggest that one or two molecules of TDdPTDA coordinated with a metal ion. The $D_{\text {Am }}$ values indicate a feasible separation of Am from Lns at TDdPTDA concentrations of above $0.02 \mathrm{M}$. A similar trend was observed with nitrobenzene (Fig. 3(b)), with $D_{\mathrm{Am}}, D_{\mathrm{Cm}}$, and $D_{\mathrm{Ln}}$ increasing as the concentration of TDdPTDA increased from 0.02 to $0.2 \mathrm{M}$. The slopes for Am and $\mathrm{Cm}$ were 1.4, and 1.5, respectively, while those for $\mathrm{La}, \mathrm{Nd}$, $\mathrm{Eu}, \mathrm{Gd}$ and Lu were 1.5, 1.1, 1.2, 1.3 and 1.5, respectively. The $D_{\text {Ln }}$ values are lower than those of Am and $\mathrm{Cm}$ and the slope suggests that one or two TDdPTDA molecules coordinated to a metal ion, consistent with results involving F-3. This consistency may be attributed similar characteristics of the diluents F-3 and nitrobenzene. The difference from F-3 and nitrobenzene is obvious with $n$-dodecane as the diluent (Fig. 3(c)). $D_{\text {Am }}$ and $D_{\mathrm{Cm}}$ increased as the concentration of TDdPTDA changed from 0.02 to $0.2 \mathrm{M}$. The slopes of the regression lines toward Am and $\mathrm{Cm}$ were 2.5. $D_{\mathrm{Ln}}$ also increased with increasing concentrations of TDdPTDA, with slopes of 2.6, 2.3, 2.5, 2.6, and 2.5, for $\mathrm{La}, \mathrm{Nd}, \mathrm{Eu}, \mathrm{Gd}$ and $\mathrm{Lu}$ respectively. The slopes were higher in the $n$-dodecane system than in the F-3 and nitrobenzene systems. This result also suggest that the number of two or three TDdPTDA molecules coordinated to a metal ion.

The effects of the nitric acid concentration on $D_{\mathrm{Ln}}, D_{\mathrm{Am}}$, and $D_{\mathrm{Cm}}$ for $0.2 \mathrm{M}$ TDdPTDA diluted by F-3, nitrobenzene, and $n$ dodecane are shown in Figs. 4(a) - 4(c). $D_{\mathrm{Am}}$ and $D_{\mathrm{Cm}}$ increased with increasing nitric acid concentration from 0.4 to $2.5 \mathrm{M}$, and decreased gradually with increasing concentration from 2.5 to $10 \mathrm{M}$ for F-3 as the diluent. The slopes for Am and $\mathrm{Cm}$ were 2.4 and 2.7, respectively, for nitric acid concentration of $0.4-$ $2.5 \mathrm{M}$. $D_{\mathrm{Ln}}$ also increased as the concentrations of nitric acid increased from $0.1 \mathrm{M}$ to $2-3 \mathrm{M}$ and decreased as the concentration further increased, except for $D_{\mathrm{Lu}}$. The slopes for $\mathrm{La}, \mathrm{Nd}, \mathrm{Eu}, \mathrm{Gd}$, and $\mathrm{Lu}$ were 2.2, 2.1, 2.1, 2.3, and 2.0, respectively, for nitric acid concentrations of $0.4-2.0 \mathrm{M}$. These results indicate that a TDdPTDA molecule coordinated to two or three nitrates. Lutetium showed a different behavior from other Lns; $D_{\mathrm{Lu}}$ increased with increasing nitric acid concentrations from 4.0 to $10 \mathrm{M}$, and it exceeded $D_{\mathrm{Am}}$ for nitric acid concentrations of $7.0-10 \mathrm{M}$. In HLLW, the amount of heavy Lns is low, with almost no Lu, and hence the continuous increase of $D_{\mathrm{Lu}}$ would be practically negligible. The largest $S F_{\mathrm{Am} / \mathrm{Ln}}$ and $S F_{\mathrm{Cm} / \mathrm{Ln}}$ were obtained at $3.0 \mathrm{M}$ nitric acid. The decrease in $D_{\mathrm{La}}, D_{\mathrm{Nd}}, D_{\mathrm{Eu}}$, and $D_{\mathrm{Gd}}$ at high nitric acid concentrations are likely caused by the protonation reaction of the $\mathrm{N}$ atom in the amide groups and in the phenanthroline frame or changes in the activities of various species. Further investigation is needed to better understand the unique behavior of $\mathrm{Lu}$ under highly acidic conditions. The response to nitrobenzene (Fig. 4(b)) resembles that for the F-3, with $D_{\text {Am }}$ and $D_{\mathrm{Cm}}$ increasing as the concentrations of nitric acid changed from 0.4 to $2.5 \mathrm{M}$, and decreasing gradually up to concentrations of $10 \mathrm{M}$, yielding slopes of 2.2 and 2.4 for $\mathrm{Am}$ and $\mathrm{Cm}$, respectively, in the range of $0.4-1.5 \mathrm{M}$ nitric acid. $D_{\mathrm{La}}, D_{\mathrm{Nd}}$, $D_{\mathrm{Eu}}$, and $D_{\mathrm{Gd}}$ also increased as the nitric acid concentrations increased from 0.2 to $2-3 \mathrm{M}$, and decreased gradually as the concentrations increased from $2-3$ to $10 \mathrm{M}$. The slopes for La, $\mathrm{Nd}, \mathrm{Eu}$, and $\mathrm{Gd}$ were 1.9, 2.0, 2.1, and 2.1, respectively, in nitric acid concentrations ranging from $0.4-1.5 \mathrm{M}$. Lutetium once again displayed a dissimilar extraction behavior from other Lns. $D_{\mathrm{Lu}}$ increased continuously with increasing nitric acid concentrations from 0.2 to $10 \mathrm{M}$, with the slope for $\mathrm{Lu}$ of 2.0 between $0.4-1.5 \mathrm{M}$ nitric acid. The use of $n$-dodecane as the diluent (Fig. 4(c)) also reveals some differences. $D_{\text {Am }}$ and $D_{\mathrm{Cm}}$ increased as nitric acid concentrations increased from 0.6 to 4.0 $\mathrm{M}$ and slightly decreased from 4.0 to $10 \mathrm{M}$. The slopes for $\mathrm{Am}$ and $\mathrm{Cm}$ were 4.3 and 4.4 , respectively, in the range of $1.0-3.0 \mathrm{M}$ nitric acid. $D_{\mathrm{La}}, D_{\mathrm{Nd}}, D_{\mathrm{Eu}}$, and $D_{\mathrm{Gd}}$ also increased with increase in the nitric acid concentration from 0.6 to $4.0 \mathrm{M}$, and decreased slightly beyond and up to 7.0 M. The decrease in $D$ s for Ans and Ln were, however, not as remarkable as for F-3 and nitrobenzene. The slopes for $\mathrm{La}, \mathrm{Nd}, \mathrm{Eu}$, and $\mathrm{Gd}$ were 4.0, 3.8, 4.4, and 4.0, respectively, for $1.0-3.0 \mathrm{M}$ nitric acid. The gradients for Ans and Lns were steeper than for F-3 and nitrobenzene. Lutetium failed to decrease as the nitric acid concentrations increased from 0.6 to $7.0 \mathrm{M}$. The slope for $\mathrm{Lu}$ of 4.7 between $1.0-3.0 \mathrm{M}$ nitric acid was steeper compared to those of the Lns. The difference in the slopes shown in Figs. 4(a) - 4(c) indicates that there is a difference in the number 


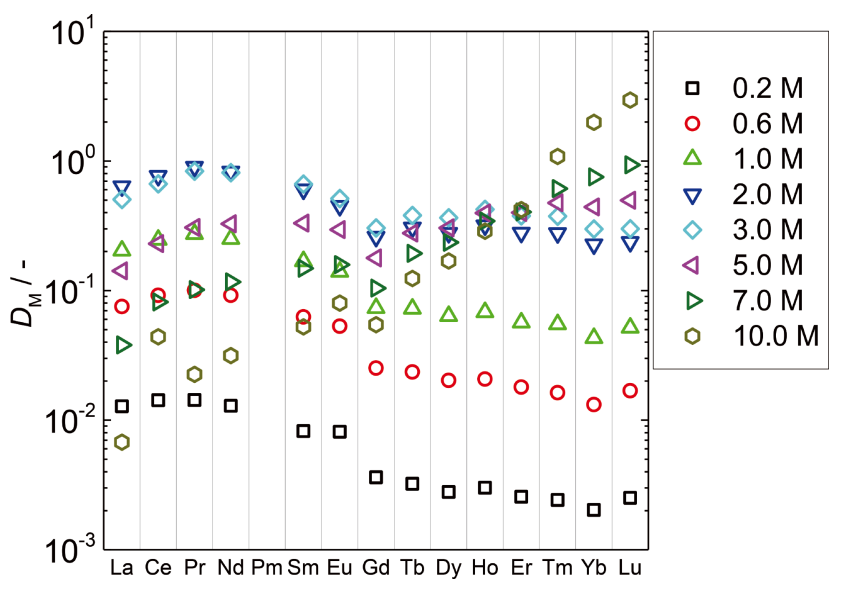

Fig. 5 Variation of $D_{\mathrm{Ln}}$ with respect to the nitric acid concentration using nitrobenzene as a diluent.

of nitrates coordinated to each metal ion, and this difference could be attributed to the polarity of each solvent. In further investigation, the ionic intensity and nitric acid uptake should be taken into account, but a rough estimation is possible.

The slopes of the extraction of Am, Cm, and Lns under the present extraction systems suggest that the solutions contain more than a single complex species, depending on the solvent. The F-3 and nitrobenzene because of high polarities facilitated the extraction of unsaturated metal-ligand complexes, like $\left[\mathrm{ML}\left(\mathrm{NO}_{3}\right)_{2}\right]^{-}$. Contrarily, the $n$-dodecane with a low polarity solvent favored extra nitrate ions accompanied by other complexes. This likely explains why the slopes of the $n$-dodecane-nitrate ion system surpass those of F-3 and nitrobenzene. To confirm the actual extracted species, other techniques including UV titration and solution EXAFS with single-crystal XRD are effective.

Figure 5 shows the variation of $D_{\mathrm{Ln}}$ with respect to nitric acid concentrations for nitrobenzene as the diluent. Significant changes were evident in the lanthanide patterns as the nitric acid concentrations increased. The $D \mathrm{~s}$ of the light and middle Lns (from $\mathrm{La}$ to $\mathrm{Ho}$ ) decreased as the concentration of nitric acid increased from 3.0 to $10 \mathrm{M}$. In contrast, the $D$ s of the heavy Lns ( $\mathrm{Tm}, \mathrm{Yb}$, and $\mathrm{Lu}$ ) increased monotonically with the increase in the nitric acid concentration from 0.2 to $10 \mathrm{M}$. This is probably because the higher surface charge density of heavy Lns produces a stronger coordination of $\mathrm{Ln}-\mathrm{O}$ (amide) more than $\mathrm{H}-\mathrm{O}$ (amide) (protonation of amide $\mathrm{O}$ ), and hence their stronger affinities toward the amide atom. ${ }^{24}$

$S F_{\mathrm{Am} / \mathrm{Ln}}$ and $S F_{\mathrm{Cm} / \mathrm{Ln}}$ for $0.2 \mathrm{M}$ of TDdPTDA and $3.0 \mathrm{M}$ of nitric acid are displayed in Figs. 6(a), 6(b), and Fig. S2 (Supporting Information). The highest $S F$ of $\mathrm{Am}$ is $S F_{\mathrm{Am} / \mathrm{Gd}}=$ 15.4, while the lowest value is $S F_{\mathrm{Am} / \mathrm{La}}=4.9$ for $\mathrm{F}-3$ as the diluent. Nitrobenzene as diluent yielded the highest $S F$ of Am as $S F_{\mathrm{Am} / \mathrm{Lu}}=18.2$ and the lowest value as $S F_{\mathrm{Am} / \mathrm{Nd}}=5.3$. The result for the F-3 and nitrobenzene diluents portrayed a similar tendency; the $S F$ s of Am for heavy Lns surpassed those of light Lns. The $S F$ s of $\mathrm{Cm}$ also exhibits a similar tendency to Am, albeit with lower values. The $S F$ s of Am with F-3 and/or nitrobenzene as diluents exceeded five that appears sufficient, whereas the $S F \mathrm{~s}$ of $\mathrm{Cm}$ require increase through further modification of the side-chain or central structure. For $n$-dodecane as the diluent, the highest $S F$ of $\mathrm{Am}$ is $S F_{\mathrm{Am} / \mathrm{Gd}}=7.9$ while the lowest value is $S F_{\mathrm{Am} / \mathrm{Yb}}=2.5$, with a discernible difference with the heavy Lns. The results for F-3 and
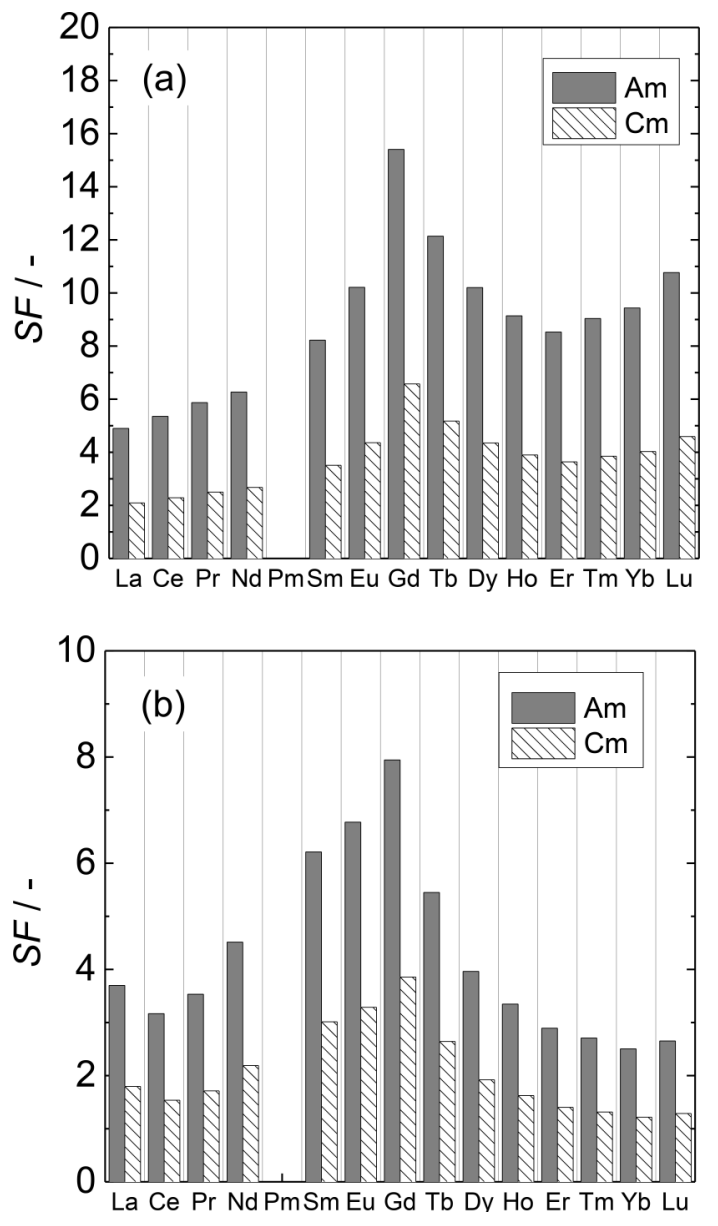

Fig. 6 (a) $S F_{\mathrm{Am} / \mathrm{Ln}}$ and $S F_{\mathrm{Cm} / \mathrm{Ln}}$ with F-3 as the diluent ([TDdPTDA] = $0.2 \mathrm{M}$ and $\left[\mathrm{HNO}_{3}\right]=3.0 \mathrm{M}$ ). (b) $S F_{\mathrm{Am} / \mathrm{Ln}}$ and $S F_{\mathrm{Cm} / \mathrm{Ln}}$ with $n$-dodecane +10 vol.\% 2-ethyl-1-hexanol as the diluent ([TDdPTDA] $=0.2 \mathrm{M}$ and $\left.\left[\mathrm{HNO}_{3}\right]=3.0 \mathrm{M}\right)$.

nitrobenzene as diluents reveal the local minima of $S F$ s for Am and $\mathrm{Cm}$ are linked to Ho or Er, but with $n$-dodecane, the $S F$ of $\mathrm{Am}$ and $\mathrm{Cm}$ decreased as the atomic number of the heavy Lns increased. The $S F$ s of Am for light Lns including $\mathrm{La}, \mathrm{Ce}, \mathrm{Pr}$, $\mathrm{Nd}$, Sm, Dy, and $\mathrm{Gd}$, were above three, suggesting easy separation of Am and light Lns. The SFs of Am for heavy Lns like Dy, Ho, Er, Tm, Yb, and Lu are lower than those observed in light Lns, indicating a rather difficult separation of Am from heavy Lns by single-stage extraction. However, the $S F$ s of 2 are enough if we use a multi-stage device, such as a mixer-settler extractor. Moreover, the amount of heavy Lns in HLLW remains low relative to light Lns, suggesting a minimal effect of heavy Lns. The lower $S F$ of $\mathrm{Cm}$ can be improved by optimizing the solution condition, like the TDdPTDA concentration, considering that $S F$ normally increases with increasing ligand concentration.

\section{Conclusions}

The general extraction behavior of MAs (Am and $\mathrm{Cm}$ ) and Lns by a novel extractant, TDdPTDA, was examined in this study. Nitric acid and ligand concentration dependencies on $D$ and $S F$ with different diluents including F-3, nitrobenzene, and $n$-dodecane were also assessed. The TDdPTDA extracted Am, $\mathrm{Cm}$, and Eu with extraction kinetics high enough for stability in a continuous counter-current extraction system. The extraction 
behavior of Am, Cm, and Lns observed in F-3 and nitrobenzene differed from those in $n$-dodecane, and this is attributed to a difference in the polarity. It was revealed that TDdPTDA had potential as the extractant for MA/Ln separation with not only nitrobenzene and F-3, but also $n$-dodecane were used as the diluents. Modification of the side-chain structure of PTDA and/ or optimization the of extraction condition produced higher $D_{\mathrm{Am}}$ and $D_{\mathrm{Cm}}$ as well as higher $S F$ of $\mathrm{Am}$ and $\mathrm{Cm}$ from Lns.

\section{Acknowledgements}

This work was supported by the internal fund in JAEA and a Grant-in-Aid for challenging Research (Exploratory, 18K19042) by the Japan Society for the Promotion Science and Joint Research with laboratory for Advanced Nuclear Energy, Tokyo Tech (2019-12).

\section{Supporting Information}

Figure S1: Contact time dependency of $D_{\mathrm{Eu}}, D_{\mathrm{Am}}$, and $D_{\mathrm{Cm}}$ for $0.2 \mathrm{M}$ TDdPTDA diluted with $n$-dodecane. Figure $\mathrm{S} 2: S F_{\mathrm{Am} / \mathrm{Ln}}$ and $S F_{\mathrm{Cm} / \mathrm{Ln}}$ with nitrobenzene as the diluent. This material is available free of charge on the Web at http://www.jsac.or.jp/ analsci/.

\section{References}

1. H. Oigawa, K. Tsujimoto, K. Nishihara, T. Sugawara, Y. Kurata, H. Takei, S. Saito, T. Sasa, and H. Obayashi, J. Nucl. Mater., 2011, 415, 245.

2. T. Sugawara, H. Takei, H. Iwamoto, A. Oizumi, K. Nishihara, and K. Tsujimoto, Prog. Nucl. Energy, 2018, 106, 27.

3. Y. Ban, H. Suzuki, S. Hotoku, T. Kawasaki, H. Sagawa, N. Tsutsui, and T. Matsumura, Solvent Extr. Ion Exch., 2019, 37, 27.

4. C. Madic, B. Boullis, P. Baron, F. Testard, M. J. Hudson, J. O. Liljenzin, B. Christiansen, M. Ferrando, A. Facchini, A. Geist, G. Modolo, A. G. Espartero, and J. De Mendoza, J. Alloys Compd., 2007, 444-445, 23.

5. J. Brown, M. J. Carrott, O. D. Fox, C. J. Maher, C. Mason, F. McLachlan, M. J. Sarsfield, R. J. Taylor, and D. A. Woodhead, IOP Conf. Ser: Mater. Sci. Eng., 2010, 9, 012075 .

6. M. Nilsson and K. L. Nash, Solvent Extr. Ion Exch., 2007, 25,665 .

7. G. Modolo, P. Kluxen, and A. Geist, Radiochim. Acta,
2010, 98, 193.

8. A. V. Gelis and G. J. Lumetta, Ind. Eng. Chem. Res., 2014, $53,1624$.

9. M. Galletta, S. Scaravaggi, E. Macerata, A. Famulari, A. Mele, W. Panzeri, F. Sansone, A. Casnati, and M. Mariani, Dalton Trans., 2013, 42, 16930.

10. M. Y. Alyapyshev, V. A. Babain, L. I. Tkachenko, A. Paulenova, A. A. Popova, and N. E. Borisova, Solvent Extr. Ion Exch., 2014, 32, 138.

11. C.-L. Xiao, C.-Z. Wang, L.-Y. Yuan, B. Li, H. He, S. Wang, Y.-L. Zhao, Z.-F. Chai, and W.-Q. Shi, Inorg. Chem., 2014, 53, 1712.

12. Y. Sasaki, Y. Tsubata, Y. Kitatsuji, and Y. Morita, Chem. Lett., 2013, 42, 91.

13. Y. Sasaki, Y. Tsubata, Y. Kitatsuji, Y. Sugo, N. Shirasu, Y. Morita, and T. Kimura, Solvent Extr. Ion Exch., 2013, 31, 401.

14. H. Suzuki, Y. Tsubata, T. Kurosawa, M. Shibata, T. Kawasaki, S. Urabe, and T. Matsumura, Anal. Sci., 2016 , 32, 477.

15. H. Suzuki, Y. Tsubata, and T. Matsumura, Anal. Sci., 2017, 33,239

16. D. Manna, S. Mula, A. Bhattacharyya, S. Chattopadhyay, and T. K. Ghanty, Dalton Trans., 2015, 44, 1332.

17. M. Alyapyshev, J. Ashina, D. Dar' in, E. Kenf, D. Kirsanov, L. Tkachenko, A. Legin, G. Starova, and V. Babain, RSC Adv., 2016, 6, 68642 .

18. N. E. Dean, R. D. Hancock, C. L. Cahill, and M. Frisch, Inorg. Chem., 2008, 47, 2000.

19. N. J. Williams, D. G. Ballance, J. H. Reibenspies, and R. D. Hancock, Inorg. Chim. Acta, 2010, 363, 3694.

20. Y. Ban, S. Hotoku, Y. Tsubata, N. Tsutsui, and T. Matsumura, Solvent Extr. Res. Dev., Jpn., 2015, 22, 47.

21. Z. Kolarik, U. Mullich, and F. Gassner, Solvent Extr. Ion Exch., 1999, 17, 1155.

22. Y. Sasaki, Y. Sugo, S. Suzuki, and S. Tachimori, Solvent Extr. Ion Exch., 2001, 19, 91.

23. A. N. Turanov, V. K. Karandashev, V. E. Baulin, A. N. Yarkevich, and Z. V. Safronova, Solvent Extr. Ion Exch., 2009, 27, 551 .

24. M. Nakase, T. Kobayashi, H. Shiwaku, T. Kawamura, and K. Takeshita, Prog. Nucl. Sci. Technol., 2018, 5, 56.

25. D. R. Raut, P. K. Mohapatra, S. A. Ansari, S. V. Godbole, M. Iqbal, D. Manna, T. K. Ghanty, J. Huskens, and W. Verboom, RSC Adv., 2013, 3, 9296.

26. M. Alyapyshev, V. Babain, I. Eliseev, E. Kenf, and L. Tkachenko, J. Radioanal. Nucl. Chem., 2016, 310, 785.

27. A. Ghanadzadeh Gilani, H. Ghanadzadeh Gilani, M. Ansari, and N. Ojani, J. Chem. Thermodyn., 2012, 44, 44. 